\title{
Stability in a Nonlinear Population Maturation Model
}

\author{
Stéphane Mischler \\ Laboratoire de Mathématiques Appliquées \\ Université de Versailles- St Quentin \\ 45, av. des Etats-Unis, 78035 Versailles, France \\ Benoît Perthame \\ Département de Mathématiques \\ et Applications, UMR8553, \\ Ecole Normale Supérieure, 45, rue d'Ulm, \\ Lenya Ryzhik \\ Department of Mathematics \\ University of Chicago, \\ Chicago IL 60637, USA
} 75230 Paris Cedex 05, France

November 26, 2004

\begin{abstract}
We consider models for population structured by maturation/maturation speed proposed by Rotenberg. It is a variant of transport equations for age-structured populations which presents particularly interesting mathematical difficulties. It allows to introduce more stochasticity in the birth process and in the aging phenomena. We present a new method for studying the time asymptotics which is also illustrated on the simpler McKendrick-Von Foerster model. The nonlinear variants of this models are shown to exhibit either nonlinear stability or periodic solutions depending on the datum.
\end{abstract}

Key words. Population aging, maturation, asymptotic analysis, transport equations.

AMS Class. Numbers. 35B40, 92B05, 35F30, 35Q80, 92D40.

\section{Contents}

1 Introduction 2

2 The linear model 3

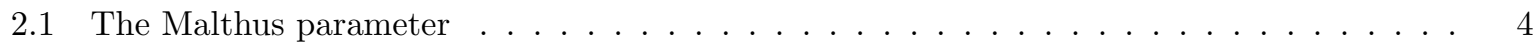

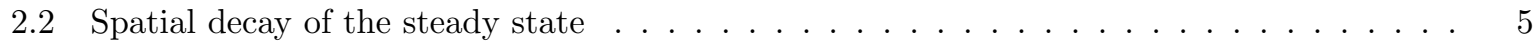

2.3 Large time asymptotics of $n$ (exponential case) $\ldots \ldots \ldots \ldots \ldots \ldots \ldots \ldots$

2.4 Time asymptotic of $n$ (general case $) \ldots \ldots \ldots \ldots \ldots$

3 Nonlinear problem $\quad 9$

3.1 A general stable case . . . . . . . . . . . . . . . . . . . . . . . . . . . 10

3.2 Periodic solutions . . . . . . . . . . . . . . . . . . . . . . . . 10

3.3 The general existence of solutions and steady state . . . . . . . . . . . . . . . . 12

4 Appendix $\quad 13$

4.1 Exponential growth and decay of population . . . . . . . . . . . . . . . . . . . 15

4.2 Equilibrium case . . . . . . . . . . . . . . . . . . . . . . 17 


\section{Introduction}

Among the wide subject of structured population dynamics, age structured models play a central role. They allow to represent the evolution of large populations through the density $n(t, a)$ of individuals with age $a$. The simpler and most famous McKendrick-Von Foerster model (see Metz et Dieckmann [13], Murray [14], Webb [15]) has been very successful in mathematical demography (see for instance the survey of Iannelli [10], the case with delay in Marcati [12] and the many references therein). It simply assumes age dependent mortality rate $D(a)$ and birth rate $B(a)$ for the population. This model, however, does not take into account aging processes which are of current biological interest for cells, microbial or virus populations. For instance, degradation can occur in the reproduction stage based on genetic degeneracy (see Edelstein-Keshet et al [9] for a general presentation of this aspect and especially for an explanation in the case of stem cells). More empirically, different individual cells, under constant environmental conditions, have been proved to exhibit highly variable intermitotic intervals (Lebowitz and Rubinow [11] and the references therein) and this observation led those authors to postulate an improved mathematical model where the population is also structured in term of a generation time $\tau$, thus leading to the Partial Differential Equation for the cell density $n(t, a, \tau)$

$$
\begin{aligned}
& \frac{\partial}{\partial t} n(t, a, \tau)+\frac{\partial}{\partial a} n(t, a, \tau)+D(a, \tau) n(t, a, \tau)=0, \quad t \geq 0, a \geq 0, \tau \geq 0, \\
& n(t, a=0, \tau)=\int b\left(a, \tau, \tau^{\prime}\right) n\left(t, a, \tau^{\prime}\right) d a d \tau^{\prime} \\
& n(t=0, a, \tau)=n_{0}(a, \tau) .
\end{aligned}
$$

This evolution equation describes aging and death of individuals, while the boundary condition at $a=$ 0 describes the birth process. Actually, the authors of [11] postulate that $B\left(a, \tau, \tau^{\prime}\right)$ concentrate on $a=\tau^{\prime}$ where the biological explanation becomes clear: $\tau$ is indeed the generation time. Notice that McKendrick-Von Foerster model (see the Appendix) is just recovered as an equation for the average $\bar{n}(t, a)=\int n(t, a, \tau) d \tau$ in the simple case where $B\left(a, \tau, \tau^{\prime}\right)=B(a)$ i.e. birth process neither alters nor improves aging properties of the new born individuals.

A somewhat related, but more general, model is that of Rotenberg [17]. Then, the biological hidden variable is a maturation velocity $\mu \in[0,1]$, and the observable state $a$ is the biological age, more relevant than the physical age, according to general theories of senescence and aging (Arking [1]), in other words, the degree of maturity (and then $a / \mu$ is the physical age in the previous models). Then, the density of population $n(t, a, \mu)$ satisfies the transport equation

$$
\frac{\partial n}{\partial t}+\mu \frac{\partial n}{\partial a}+D(a, \mu) n=\int K\left(a, \mu, \mu^{\prime}\right) n\left(t, a, \mu^{\prime}\right) d \mu^{\prime},
$$

with again boundary conditions at $a=0$, and initial data at $t=0$,

$$
\begin{gathered}
n(t, a=0, \mu)=G_{0}(\mu) \int b\left(a^{\prime}, \mu^{\prime}\right) n\left(t, a^{\prime}, \mu^{\prime}\right) d \mu^{\prime} d a^{\prime}, \\
n(t=0, a, \mu)=n_{0}(a, \mu) .
\end{gathered}
$$

This model enhances the stochasticity of the time evolution of the population thanks to the kernel $K$ which allows a random change of the maturation velocity, and also, as in the previous models, in the birth process. This feature seems essential to explain experimentally observed deviations from the usual Gompertz law for mortality rates, see Weitz and Frazer [16].

We would like to point out that many other questions related to multi-structured population dynamics have been raised in the recent literature, especially existence of the steady states, their stability, and possible chaotic behavior of solutions. We refer, for instance, to Diekmann et al [7], Dyson et al [8] and references therein.

Our purpose in this paper is to study several aspects of these models and of some natural nonlinear versions. Since the mathematical structure in Rotenberg's model is more interesting we will essentially 
focus on that model. In the second section we study the transport equation and exhibit a Malthus parameter in the case of expanding populations and show the existence of an exponential profile i.e. a steady state for the corresponding damped equation. We also study this steady state in detail, and especially the age profile. The third section is devoted to the study of nonlinear effects which can be interpreted as competition for resources. Even though we exhibit a quite general class of examples where the steady states are nonlinearly stable, it turns out that the introduction of a global population based pressure on reproduction can lead to a purely oscillatory behavior. We use methods relying on tools introduced for transport equations by Bensoussan et al [3], Bardos et al [2] rather than the usual Laplace transform methods that are not so well suited for our models. In order to make them more transparent on a simple example, we treat in an appendix the McKendrick-Von Foerster model with this tool.

Acknowledgment. The first two authors wish to thank R. Ferrière and G. Paul for many discussions and biological references. L. Ryzhik was supported in part by NSF grant DMS-9971742, and Alfred P. Sloan Foundation.

\section{The linear model}

We consider first the linear model for the density $n(t, a, \mu)$ of population, of age $a \geq 0$ and maturation rate $\mu \in[0,1]$. The function $n$ satisfies the linear transport equation

$$
\frac{\partial n}{\partial t}+\mu \frac{\partial n}{\partial a}+D(a, \mu) n=\int K\left(a, \mu, \mu^{\prime}\right) n\left(t, a, \mu^{\prime}\right) d \mu^{\prime}
$$

with the boundary condition

$$
n(t, a=0, \mu)=G_{0}(\mu) \int b\left(a^{\prime}, \mu^{\prime}\right) n\left(t, a^{\prime}, \mu^{\prime}\right) d \mu^{\prime} d a^{\prime},
$$

and the initial data $n(0, a, \mu)=n_{0}(a, \mu) \geq 0$. Here $G_{0}(\mu)$ is a fixed distribution of the maturation rates among the new-born population,

$$
G_{0}(\mu) \geq 0, \quad G_{0}(\mu) \in L^{\infty}([0,1]),
$$

$b(a, \mu)$ is the rate at which species of age $a$ and maturation velocity $\mu$ give birth. The non-negative function $K$ describes the probability of a change of maturation rate form $\mu^{\prime}$ to $\mu$, and the function $D$ accounts both for transition from maturation rate $\mu$ to other maturation rates, and for the death rate. We assume that $D(\mu, a)$ is continuous and positive, and the birth rate $b(a, \mu)$ and the symmetric kernel $K\left(a, \mu, \mu^{\prime}\right)$ are continuous and bounded:

$$
\begin{aligned}
& 0<D(a, \mu)<+\infty, \quad 0 \leq b(a, \mu), K\left(a, \mu, \mu^{\prime}\right) \leq C, \quad \int b(a, \mu) d a d \mu<+\infty \\
& K\left(a, \mu, \mu^{\prime}\right)=K\left(a, \mu^{\prime}, \mu\right), \quad 0<\sigma_{0} \leq D(a, \mu)-\int K\left(a, \mu^{\prime}, \mu\right) d \mu^{\prime}
\end{aligned}
$$

so that in the absence of reproduction the total population vanishes exponentially fast in time.

Equation (2.1) has the form of the linear transport equation encountered, for example, in the theory of neutron scattering, or multiple scattering of waves, where $\mu$ is particle velocity and takes values in $[-1,1]$. One of the main features of (2.1) in the context of population dynamics is that maturation velocity $\mu \geq 0$ is non-negative. This allows to simplify the analysis significantly. In particular, we are interested in the existence of the Malthus parameter $\gamma$ such that $e^{-\gamma t} n(t, a)$ remains bounded, and also in the precise large time asymptotics of such quantity. 


\subsection{The Malthus parameter}

In order to study the growth or decay in time of solutions of the coupled system (2.1)-(2.2), we first look for special solutions of the form $n(t, a, \mu)=e^{\gamma t} g(a, \mu)$. The function $g$ is then an eigenvector of the corresponding stationary equation, and thus should satisfy

$$
\begin{aligned}
& \mu \frac{\partial g}{\partial a}+[D(a, \mu)+\gamma] g=\int K\left(a, \mu, \mu^{\prime}\right) g\left(a, \mu^{\prime}\right) d \mu^{\prime} \\
& g(a=0, \mu)=G_{0}(\mu)
\end{aligned}
$$

with a normalization condition

$$
\int b\left(a^{\prime}, \mu^{\prime}\right) g\left(a^{\prime}, \mu^{\prime}\right) d \mu^{\prime} d a^{\prime}=1
$$

that determines the eigenvalue $\gamma$ (the Malthus parameter). A sufficient condition for the existence of the eigenvalue $\gamma$ is given in the following lemma.

We recall that, for $\gamma \geq-\sigma_{0}$ there exists a unique bounded solution $g_{\gamma}$ to $(2.5), 0 \leq g_{\gamma} \leq\left\|G_{0}\right\|_{L^{\infty}}$, thanks to the classical theory of evolution PDEs (Dautray and Lions [6]).

Lemma 2.1 Assume (2.3), (2.4) and

$$
1 \leq \int b(a, \mu) g_{-\sigma_{0}}(a, \mu) d a d \mu<+\infty,
$$

here the function $g_{\gamma}$ denotes the solution to (2.5). Then, there is a unique $\gamma \geq-\sigma_{0}$ so that a solution to (2.5)-(2.6) exists.

Proof. Let $\gamma>\tilde{\gamma}>-\sigma_{0}$, then we have thanks to the maximum principle,

$$
0<g_{\gamma}(a, \mu)<g_{\tilde{\gamma}}(a, \mu)<g_{-\sigma_{0}}(a, \mu)<\left\|G_{0}\right\|_{L^{\infty}} .
$$

The last inequality being a consequence of the fact that $\left\|G_{0}\right\|_{L^{\infty}}$ is a super-solution to equation (2.5) for all $\gamma \geq-\sigma_{0}$. Furthermore, we can define the function

$$
I(\gamma):=\int b(a, \mu) g_{\gamma}(a, \mu) d a d \mu \geq 0
$$

Thanks to assumption (2.7) and the above inequalities, we have that $I(\gamma)$ is finite and is decreasing. Also $g_{\gamma}(a, \mu)$ depends continuously on $\gamma$ (locally in $a$ ) therefore, thanks to the Lebesgue dominated convergence theorem, $I(\gamma)$ is continuous. We know, still from $(2.7)$, that $I\left(-\sigma_{0}\right) \geq 1$. It remains to show that as $\gamma \rightarrow+\infty, I(\gamma) \rightarrow 0$. To do that, we notice that $g_{\gamma}(a, \mu) \leq \tilde{g}_{\gamma}(a, \mu)$, with

$$
\begin{aligned}
& \mu \frac{\partial \tilde{g}_{\gamma}}{\partial a}+[D(a, \mu)+\mu \gamma] \tilde{g}_{\gamma}=\int K\left(a, \mu, \mu^{\prime}\right) \tilde{g}_{\gamma}\left(a, \mu^{\prime}\right) d \mu^{\prime} \\
& \tilde{g}_{\gamma}(a=0, \mu)=G_{0}(\mu) .
\end{aligned}
$$

And one also has, still using the maximum principle, that $\tilde{g}_{\gamma}(a, \mu) \leq g_{0}(a, \mu) e^{-\gamma a}$. Therefore, as $\gamma \rightarrow \infty$,

$$
I(\gamma) \leq \int b(a, \mu) g_{0}(a, \mu) e^{-\gamma a} d a d \mu \rightarrow 0
$$

by Lebesgue dominated convergence theorem. This proves that (2.6) holds for some $\gamma \geq-\sigma_{0}$. 


\subsection{Spatial decay of the steady state}

We will assume below that (2.7) holds. If this condition is violated so that the birth rate is insufficient to sustain growth, then solution of the evolution problem (2.1)-(2.2) decays exponentially in time. We are going now to describe asymptotic properties in $a$ for the solution to (2.5)- (2.6).

We may assume without loss of generality that $\gamma=0$ is the eigenvalue given by Lemma 2.1. Otherwise, if $\gamma>-\sigma_{0}$ we may redefine $n^{\prime}(t, a, \mu)=n(t, a, \mu) e^{\gamma t}$. The function $n^{\prime}$ satisfies (2.1)-(2.2) with the modified extinction rate $D^{\prime}=D-\gamma$, and the corresponding eigenvalue $\gamma^{\prime}=0$.

We study now the asymptotic behavior of the steady state $g_{0}(a, \mu)$ in the case when the extinction rate $D(a)=d$ is a constant, and the kernel $K\left(a, \mu, \mu^{\prime}\right)=k\left(\mu, \mu^{\prime}\right) \geq k_{0}>0$ is independent of $a$ and positive. We define an operator

$$
\mathcal{K} g(\mu)=\int k\left(\mu, \mu^{\prime}\right) g\left(\mu^{\prime}\right) d \mu^{\prime}
$$

and consider an auxiliary eigenvalue problem in $C[0,1]$ for $b \geq 0$

$$
\frac{1}{d-b \mu} \mathcal{K} \psi(\mu)=\lambda(b) \psi(\mu) \text {. }
$$

A positive eigenfunction $\psi(\mu)>0$ exists for each $b$ according to the Krein-Rutman theorem. Integrating (2.10) in $\mu$ we obtain $1-\sigma_{0} / d \leq \lambda(0)<1$, while

$$
\lambda(b) \geq \frac{k_{0}}{b} \ln \frac{d}{d-b}
$$

blows up as $b \rightarrow d$. Therefore there exists $0<b_{0}<d$ such that $\lambda\left(b_{0}\right)=1$ and the corresponding eigenfunction satisfies

$$
-b_{0} \mu \psi(\mu)+d \psi(\mu)=\int k\left(\mu, \mu^{\prime}\right) \psi\left(\mu^{\prime}\right) d \mu^{\prime} .
$$

We will normalize the function $\psi$ so that

$$
\int \mu|\psi(\mu)|^{2} d \mu=1
$$

Furthermore, since $\psi(\mu)>0$ there exists a constant $C>0$ so that $G_{0}(\mu) \leq C \psi(\mu)$. The comparison principle implies that

$$
g_{0}(a, \mu) \leq C \psi(\mu) e^{-b_{0} a} .
$$

We can now state our second result which makes more precise the asymptotic behavior of $g_{0}$.

Lemma 2.2 With the above notation, and for $\gamma=0$ achieved in Lemma 2.1, $g_{0}(a, \mu)$ can be written as

$$
g_{0}(a, \mu)=Q(a, \mu) \psi(\mu) e^{-b_{0} a}, \quad 0<b_{0}<d .
$$

Moreover, let the constant $q_{\infty}$ be defined by

$$
q_{\infty}=\int \mu Q(a=0, \mu)|\psi(\mu)|^{2} d \mu=\int \mu G_{0}(\mu)|\psi(\mu)| d \mu
$$

then there exist constants $\alpha>0$ and $C>0$ so that we have $Q=q(a)+w(a, \mu)$ and

$$
\int e^{2 \alpha a}\left|q(a)-q_{\infty}\right|^{2} d a+\int e^{2 \alpha a}|w(a, \mu)|^{2} d \mu d a \leq C .
$$


Proof. As a first step, we write the equation on $Q$. It satisfies

$$
\begin{aligned}
& \mu \frac{\partial Q(a, \mu)}{\partial a}+\left[d-b_{0} \mu\right] Q(a, \mu)=\int k\left(\mu, \mu^{\prime}\right) \frac{\psi\left(\mu^{\prime}\right)}{\psi(\mu)} Q\left(a, \mu^{\prime}\right) d \mu^{\prime}, \\
& Q(a=0, \mu)=Q_{0}(\mu)=\frac{G_{0}(\mu)}{\psi(\mu)},
\end{aligned}
$$

and is uniformly bounded as follows from (2.11). We define the operator $\mathcal{L}$ by

$$
\mathcal{L} f(\mu)=\int k\left(\mu, \mu^{\prime}\right) \frac{\psi\left(\mu^{\prime}\right)}{\psi(\mu)} f\left(\mu^{\prime}\right) d \mu^{\prime}+b_{0} \mu f(a, \mu) .
$$

The constant function $f=1$ is the eigenfunction of $\mathcal{L}$ corresponding to the eigenvalue $\lambda=d: \mathcal{L} 1=d \cdot 1$. The operator $\mathcal{L}$ is self-adjoint in the space $L_{\psi}^{2}[0,1]$ with the inner product

$$
\langle f, g\rangle_{\psi}=\int f(\mu) \bar{g}(\mu)|\psi(\mu)|^{2} d \mu .
$$

The Krein-Rutman theorem implies that it has spectral radius $d$, and $d$ is an isolated eigenvalue. Therefore there exists a constant $\alpha_{0}$ so that

$$
\langle(d-\mathcal{L}) w, w\rangle_{\psi} \geq \alpha_{0}\|w\|_{L_{\psi}^{2}[0,1]}^{2},
$$

provided that $\langle w, 1\rangle_{\psi}=0$. We may decompose $Q=w(a, \mu)+q(a)$, with $\int w(a, \mu)|\psi(\mu)|^{2} d \mu=0$.

In a second step, we prove that $q(a)$ converges to a constant $q_{\infty}$ as $a \rightarrow 0$, and $w(a, \mu)$ goes to zero exponentially fast. We write $Q=q_{\infty}+Q^{\prime}(a, \mu)$, so that the function $Q^{\prime}$ satisfies $(2.13)$ with the modified initial data $Q^{\prime}(0, \mu)=Q_{0}(\mu)-q_{\infty}$, and in particular

$$
\int \mu Q^{\prime}(0, \mu)|\psi(\mu)|^{2} d \mu=0 .
$$

This implies that

$$
\int \mu Q^{\prime}(a, \mu)|\psi(\mu)|^{2} d \mu=0 \text { for all } a \geq 0 .
$$

We decompose $Q^{\prime}(a, \mu)=q^{\prime}(a)+w(a, \mu)$ with $q^{\prime}(a)=q(a)-q_{\infty}$. Then we have from (2.15) for all $a \geq 0$ :

$$
q^{\prime}(a)=-\int \mu w(a, \mu)|\psi(\mu)|^{2} d \mu
$$

and thus, by Cauchy-Schwartz inequality,

$$
\left|q^{\prime}(a)\right| \leq C\left(\int w^{2}(\mu) d \mu\right)^{1 / 2} .
$$

Next we multiply equation (2.13) for $Q^{\prime}$ by $Q^{\prime} e^{2 \alpha a}$ with the constant $\alpha$ to be determined and obtain

$$
\frac{1}{2} \mu \frac{\partial}{\partial a}\left(e^{\alpha a} Q^{\prime}\right)^{2}+Q^{\prime} e^{\alpha a} \mathcal{L}\left[w e^{\alpha a}\right]=\alpha \mu\left(Q^{\prime} e^{\alpha a}\right)^{2} .
$$

We integrate this equation in $\mu$ and use (2.14) to obtain

$$
\frac{1}{2} \frac{\partial}{\partial a} \int \mu\left(e^{\alpha a} Q^{\prime}\right)^{2} d \mu+\alpha_{0} e^{2 \alpha a} \int w^{2}(a, \mu) d \mu \leq 2 e^{2 \alpha a} \int \alpha \mu\left(w^{2}+\left(q^{\prime}\right)^{2}\right) d \mu .
$$


We use the bound (2.16) to conclude that for sufficiently small $\alpha>0$ we have

$$
\frac{1}{2} \int e^{2 \alpha a} Q^{2}(a, \mu) d \mu+C \int w^{2}(a, \mu) e^{2 \alpha a} d a d \mu \leq \frac{1}{2} \int Q_{0}^{2}(\mu) d \mu
$$

and in particular

$$
\int w^{2}(a, \mu) e^{2 \alpha a} d a d \mu \leq C .
$$

Now (2.12) follows from (2.16) and (2.17).

One may improve the $L^{2}$-exponential convergence to a constant in Lemma 2.2 to uniform convergence, as in [2], or using the probabilistic techniques of [3], but we do not dwell on this here.

\subsection{Large time asymptotics of $n$ (exponential case)}

The next proposition shows that $n(t, a, \mu)$ converges as $t \rightarrow+\infty$ to a multiple of the steady state $g_{0}(a, \mu)$ (we still assume without further restriction that $\gamma=0$ is achieved in Lemma 2.1). First we need to define an auxiliary function $H(a, \mu)$ that solves the dual problem to (2.5)-(2.6), namely

$$
\begin{aligned}
& \mu \frac{\partial H}{\partial a}-D(a, \mu) H=-\int K\left(a, \mu^{\prime}, \mu\right) H\left(a, \mu^{\prime}\right) d \mu^{\prime}-b(a, \mu), \\
& \int \mu G_{0}(\mu) H(0, \mu)=1, \\
& H(a, \mu) \rightarrow 0 \text { as } a \rightarrow \infty .
\end{aligned}
$$

Existence of a non-negative solution to (2.18) is shown as follows. We let $H_{n}(a, \mu)=0$ for $a \geq n$, while on the interval $0 \leq a \leq n$ the function $H_{n}$ is the solution of

$$
\begin{aligned}
& \mu \frac{\partial H_{n}}{\partial a}-D(a, \mu) H_{n}=-\int K\left(a, \mu^{\prime}, \mu\right) H_{n}\left(a, \mu^{\prime}\right) d \mu^{\prime}-b(a, \mu) \chi\left(\frac{a}{n}\right), \\
& H_{n}(n, \mu)=0 .
\end{aligned}
$$

The function $\chi(a)$ is monotonically decreasing and smooth, and satisfies

$$
\chi(a)= \begin{cases}1, & a \leq 1 / 2 \\ 0, & a \geq 1\end{cases}
$$

The sequence $H_{n}(a, \mu) \geq 0$ is point-wise monotonically increasing to a limit $H(a, \mu)$ as $n \rightarrow \infty$. Integrating (2.19) we obtain

$$
\int \mu H_{n}(0, \mu) d \mu+\sigma_{0} \int H_{n}(a, \mu) d a d \mu \leq \int b(a, \mu) d a d \mu,
$$

so that $H_{n}$ is a bounded sequence in $L^{1}$, and thus the limit $H(a, \mu) \geq 0$ belongs to $L^{1}$. The function $H$ satisfies the first and third equations in the dual problem (2.18). Furthermore, multiplying (2.18) by $g_{0}(a, \mu)$ we obtain

$$
\int \mu g_{0}(0, \mu) H(0, \mu) d \mu=\int b(a, \mu) g_{0}(a, \mu) d a d \mu .
$$

Using the normalization condition (2.6) we obtain the second condition in (2.18). The following proposition characterizes convergence of the solution of the time-dependent problem to the steady state. 
Proposition 2.3 Under the assumptions of Lemma 2.1, with the value $\gamma=0$ achieved in there, assume that for some $\lambda \geq 0$ we have $b(a, \mu) \geq \lambda H(a, \mu)$ and let $n(t, a, \mu)$ be the solution of the initial value problem (2.1)-(2.2) with the initial data $n_{0}(a, \mu) \in L^{1} \cap L^{\infty}\left(\mathbb{R}_{+} \times[0,1]\right)$. Then we have

$$
\int H(a, \mu)\left|n(t, a, \mu)-\beta g_{0}(a, \mu)\right| d a d \mu \leq e^{-\lambda t} \int H(a, \mu)\left|n_{0}(a, \mu)-\beta g_{0}(a, \mu)\right| d a d \mu .
$$

The constant $\beta$ is given by $\beta=\frac{\int n_{0}(a, \mu) H(a, \mu) d a d \mu}{\int g_{0}(a, \mu) H(a, \mu) d a d \mu}$.

Proof. We let $\tilde{n}(t, a, \mu)=n(t, a, \mu)-\beta g(a, \mu)$ so that the function $\tilde{n}$ satisfies (2.1)-(2.2) with the initial data $\tilde{n}_{0}(a, \mu)=n_{0}(a, \mu)-\beta g(a, \mu)$. Then we have for all $t \geq 0$ :

$$
\int \tilde{n}(t, a, \mu) H(a, \mu) d a d \mu=0 .
$$

The modulus $|\tilde{n}|$ satisfies

$$
\begin{aligned}
\frac{\partial|\tilde{n}|}{\partial t} & +\mu \frac{\partial|\tilde{n}|}{\partial a}+D|\tilde{n}| \leq \int k\left(\mu, \mu^{\prime}\right)\left|\tilde{n}\left(\mu^{\prime}\right)\right| d \mu^{\prime} \\
|\tilde{n}|(t, a=0, \mu) & =G_{0}(\mu)\left|\int b(a, \mu) \tilde{n}(t, a, \mu) d a d \mu\right| \\
& =G_{0}(\mu)\left|\int(b(a, \mu)-\lambda H(a, \mu)) \tilde{n}(t, a, \mu) d a d \mu\right| \\
& \leq G_{0}(\mu) \int(b(a, \mu)-\lambda H(a, \mu))|\tilde{n}(t, a, \mu)| d a d \mu .
\end{aligned}
$$

Multiplying equation $(2.22)$ by $H(a, \mu)$ we obtain from the above calculation

$$
\begin{aligned}
\frac{d}{d t} \int H(a, \mu)|\tilde{n}(t, a, \mu)| d a d \mu & \leq \int \mu|\tilde{n}(t, a=0, \mu)| H(0, \mu) d \mu-\int b(a, \mu)|\tilde{n}(t, a, \mu)| d a d \mu \\
& \leq\left|\int b(a, \mu) \tilde{n}(t, a, \mu) d a d \mu\right|-\int b(a, \mu)|\tilde{n}(t, a, \mu)| d a d \mu \\
& \left.\leq-\lambda \int H(a, \mu)\right)|\tilde{n}(t, a, \mu)| d a d \mu .
\end{aligned}
$$

The result of Proposition 2.3 follows by Gronwall lemma.

\subsection{Time asymptotic of $n$ (general case)}

The time asymptotics in Proposition 2.3 requires the assumption that $b(a, \mu) \geq \lambda H(a, \mu)$. As shown explicitly in the appendix, this is somewhat restrictive close to $a=0$ where $b(a, \mu)$ cannot vanish. We provide a more general result which uses the notations of Proposition 2.3.

Proposition 2.4 Under the assumptions of Lemma 2.1, with the value $\gamma=0$ achieved in there, let $n(t, a, \mu)$ be the solution of the initial value problem (2.1)-(2.2) with the initial data $n_{0}(a, \mu) \leq C g_{0}(a, \mu)$. Then we have

$$
\int H(a, \mu)\left|n(t, a, \mu)-\beta g_{0}(a, \mu)\right| d a d \mu \rightarrow 0 \text { as } t \rightarrow+\infty \text {. }
$$


Proof. 1st step. Construction of a limit $m(t, a, \mu)$. Following the above proof, we arrive at

$$
\frac{d}{d t} \int H(a, \mu)|\tilde{n}(t, a, \mu)| d a d \mu \leq\left|\int b(a, \mu) \tilde{n}(t, a, \mu) d a d \mu\right|-\int b(a, \mu)|\tilde{n}(t, a, \mu)| d a d \mu .
$$

Therefore, $P(t)=\int H(a, \mu)|\tilde{n}(t, a, \mu)| d a d \mu$ is a decreasing function of time, and the $\operatorname{limit}_{\infty}=\lim _{t \rightarrow \infty} P(t) \geq$ 0 exists. We claim that $P_{\infty}=0$. Indeed, as a consequence of (2.23), we have

$$
\int_{0}^{\infty}\left[\int b(a, \mu)|\tilde{n}(t, a, \mu)| d a d \mu-\left|\int b(a, \mu) \tilde{n}(t, a, \mu) d a d \mu\right|\right] d t \leq P(0) .
$$

Furthermore, we have $\left|\tilde{n}_{0}(a, \mu)\right| \leq C g_{0}(a, \mu)$ and thus $|\tilde{n}(t, a, \mu)| \leq C g_{0}(a, \mu)$ and is uniformly bounded for all times. Therefore, we may choose a sequence of times $t_{k} \rightarrow+\infty$ so that the sequence of functions $\tilde{n}_{k}(t, a, \mu)=\tilde{n}\left(t_{k}+t, a, \mu\right)$ converges weak-* in $L^{\infty}((0, T) \times(0, \infty) \times(0,1))$, for any $T>0$, to a function denoted $m(t, a, \mu)$. From (2.24), we deduce

$$
\int_{0}^{T}\left[\int b(a, \mu)\left|\tilde{n}_{k}(t, a, \mu)\right| d a d \mu-\left|\int b(a, \mu) \tilde{n}_{k}(t, a, \mu) d a d \mu\right|\right] d t \rightarrow 0 \quad \text { as } k \rightarrow \infty .
$$

2nd step. Properties of $m(t, a, \mu)$. For any smooth function $\varphi(a, \mu)$, and using equation (2.1), we have that

$$
\frac{d}{d t} \int \tilde{n}_{k}(t, a, \mu) \varphi(a, \mu) d a d \mu \quad \text { is bounded in } L^{\infty}(0, \infty)
$$

Therefore, after approximating $b$ by smooth functions, we deduce that $\int b(a, \mu) \tilde{n}_{k}(t, a, \mu) d a d \mu$ converges in $C([0, T])$ to $\int b(a, \mu) m(t, a, \mu) d a d \mu$. Passing to the limit $k \rightarrow \infty$ in $(2.26)$ we obtain

$$
\int_{0}^{T} \int b(a, \mu)\left|\tilde{n}_{k}(t, a, \mu)\right| d a d \mu \rightarrow \int_{0}^{T} \int b(a, \mu)|m(t, a, \mu)| d a d \mu .
$$

Next, using this and (2.25) we obtain by convexity,

$$
\int_{0}^{T}\left[\int b(a, \mu)|m(t, a, \mu)| d a d \mu-\left|\int b(a, \mu) m(t, a, \mu) d a d \mu\right|\right] d t \leq 0 \quad \text { and thus }=0 .
$$

This equality proves that the function $m$ has a constant sign on the support of $b$. This concludes the proof when $\operatorname{supp}(b)$ contains $\operatorname{supp}(H)$ thanks to $(2.21)$ which also gives

$$
\int m(t, a, \mu) H(a, \mu) d a d \mu=0 .
$$

Indeed, this implies $m=0$ which implies, from (2.27), strong convergence and thus the proof of Proposition 2.4. We refer to the appendix for the extension of this proof to the general case.

\section{Nonlinear problem}

Several nonlinear versions of age structured models have been proposed and studied in the past. Existence of solutions for instance was proved by Chipot [4]. Here we study more qualitative properties and try to understand whether the asymptotic convergence toward a steady state still holds for the nonlinear equation. 


\subsection{A general stable case}

Here we restrict our attention to the following non-linear version of the McKendrick-Von Foerster model

$$
\begin{aligned}
& \frac{\partial}{\partial t} n(t, a)+\frac{\partial}{\partial a} n(t, a)+D n(t, a)=0, \quad t \geq 0, a \geq 0 \\
& n(t, a=0)=\tau(N(t)) \int B(a) n(t, a) d a \\
& N(t)=\int n(t, a) d a \\
& n(t=0, a)=n_{0}(a) .
\end{aligned}
$$

The function $\tau(N)$ is assumed to be smooth and decreasing to zero as $N \rightarrow+\infty$ (the total size of the population introduces a competition for resources which induces a pressure on birth).

In order to conduct explicit calculations, we choose exponential birth rate and constant mortality rate and assume that the linear model with $\tau=\tau(0)$ gives a growing population

$$
B(a)=e^{-r a}, \quad D+r<\tau(0) .
$$

We also use the notation $\beta(t)=\int B(a) n(t, a) d a$. After integrating the equation on $n$ we obtain a coupled system of two equations

$$
\begin{aligned}
& \frac{d}{d t} N(t)=-D N(t)+\tau(N(t)) \beta(t), \\
& \frac{d}{d t} \beta(t)=(\tau(N(t))-D-r) \beta(t) .
\end{aligned}
$$

The non-trivial steady state is given by the unique solution of the two equalities

$$
\tau\left(N_{\text {crit }}\right)=D+r, \quad \beta_{\text {crit }}=D N_{\text {crit }} / \tau\left(N_{\text {crit }}\right) .
$$

The linearized matrix at the equilibrium point is given by

$$
\mathcal{L}_{\text {crit }}=\left(\begin{array}{cc}
-D+\tau^{\prime}\left(N_{\text {crit }}\right) \beta_{\text {crit }} & \tau\left(N_{\text {crit }}\right) \\
\tau^{\prime}\left(N_{\text {crit }}\right) \beta_{\text {crit }} & 0
\end{array}\right)
$$

We observe that $\operatorname{tr}\left(\mathcal{L}_{\text {crit }}\right)<0$ and $\operatorname{det}\left(\mathcal{L}_{\text {crit }}\right)>0\left(\right.$ since $\left.\tau^{\prime}<0\right)$. Therefore the two eigenvalues have negative real parts and thus the non-trivial critical point is indeed attractive.

This situation is preserved by small variations of the data and seems thus rather generic.

\subsection{Periodic solutions}

It is however possible to find cases where the same type of nonlinear model exhibits periodic solutions. We need however more elaborate data. Therefore we choose

$$
\begin{aligned}
& \frac{\partial}{\partial t} n(t, a)+\frac{\partial}{\partial a} n(t, a)=0, \quad t \geq 0, a \geq 0, \\
& n(t, a=0)=\tau(\tilde{N}(t)) \int B(a) n(t, a) d a, \\
& \tilde{N}(t)=\int \omega(a) n(t, a) d a .
\end{aligned}
$$

Here $\omega \geq 0$ denotes a weight function which allows to refine modeling of total population pressure on birth.

We are going to consider periodic solutions that are small perturbations around the steady state $n_{s t}=1$, which is achieved after renormalizing the data so that

$$
1=\tau\left(\int \omega(a) d a\right) \int B(a) d a .
$$

We look for periodic solutions in the form

$$
n_{p e r}=1+\varepsilon \cos \nu(t-a),
$$


and show that such solutions might exist. We assume for simplicity that $\tau$ is linear around the state $n=1$ so that

$$
\tau\left(\int \omega(a) d a+x\right)=\tau\left(\int \omega(a) d a\right)-\lambda_{1} x
$$

for sufficiently small $x$. We also assume that there exists $\nu \in \mathbb{R}$ that satisfies

$$
\int B(a) \sin \nu a d a=\int B(a) \cos \nu a d a=0,
$$

which means that the Fourier transform of $B(a)$ vanishes at some point. This implies that

$$
\int B(a) n_{p e r} d a=\int B(a) d a
$$

is independent of time. Therefore in order to verify that $n_{\text {per }}$ given by (3.5) satisfies (3.4) we only need to check that

$$
\cos (\nu t)=-\lambda_{1} \int \omega(a) \cos (\nu(t-a)) d a .
$$

This condition is fulfilled by the choice of $\lambda_{1}:\left(\lambda_{1}\right)^{-1}=-\int \omega(a) \cos (\nu a) d a>0$ (which is always possible) provided that $\int \omega(a) \sin (\nu a) d a=0$.

Since Rotenberg's model introduces more randomness and diffusion, it is interesting to check that such periodic solutions exist for the more general case of equation

$$
\begin{aligned}
& \frac{\partial n}{\partial t}+\mu \frac{\partial n}{\partial a}+\sigma n=0, \\
& n(t, a=0, \mu)=G_{0}(\mu) \int b\left(a^{\prime}, \mu^{\prime}\right) n\left(t, a^{\prime}, \mu^{\prime}\right) d a^{\prime} d \mu^{\prime} \tau\left(\int \omega\left(a^{\prime}, \mu^{\prime}\right) n\left(t, a^{\prime}, \mu^{\prime}\right) d a^{\prime} d \mu^{\prime}\right),
\end{aligned}
$$

when maturation rate is not restricted to a single value. A steady state solution $G(a, \mu)$ for (3.7) exists provided that

$$
I=\int b(a, \mu) G_{0}(\mu) e^{-\sigma a / \mu} d a d \mu>\frac{1}{\tau(0)}
$$

and is given by $n(a, \mu)=m G_{0}(\mu) \exp \left(-\frac{\sigma a}{\mu}\right)$. The number $m$ is the solution of

$$
\tau\left(m \int \omega(a, \mu) G_{0}(\mu) e^{-\sigma a / \mu} d a d \mu\right)=\frac{1}{I} .
$$

We look for solutions in the form

$$
n(t, a, \mu)=\phi\left(t-\frac{a}{\mu}, \mu\right) e^{-\sigma a} .
$$

We obtain from the boundary condition at $a=0$ :

$$
\phi(t, \mu)=G_{0}(\mu) \int b\left(a, \mu^{\prime}\right) \phi\left(t-\frac{a}{\mu^{\prime}}, \mu^{\prime}\right) e^{-\sigma a} d a d \mu^{\prime} \tau\left(\int \omega\left(a, \mu^{\prime}\right) \phi\left(t-\frac{a}{\mu^{\prime}}, \mu^{\prime}\right) e^{-\sigma a} d a d \mu^{\prime}\right) .
$$

In order to be as explicit as possible we look for a perturbation of a steady state of the form

$$
\phi(t, \mu)=m G_{0}(\mu)[1+\varepsilon \zeta(t)] .
$$


This implies that

$$
\begin{aligned}
& 1+\varepsilon \zeta(t)=\int b\left(a, \mu^{\prime}\right) G_{0}\left(\mu^{\prime}\right)\left(1+\varepsilon \zeta\left(t-\frac{a}{\mu^{\prime}}\right)\right) e^{-\sigma a} d a d \mu^{\prime} \\
& \times \tau\left(m \int \omega\left(a, \mu^{\prime}\right) G_{0}\left(\mu^{\prime}\right) e^{-\sigma a} d a d \mu+m \varepsilon \int \omega\left(a, \mu^{\prime}\right) G_{0}\left(\mu^{\prime}\right) \zeta\left(t-\frac{a}{\mu^{\prime}}\right) e^{-\sigma a} d a d \mu^{\prime}\right) .
\end{aligned}
$$

As before, in order to avoid technicalities we will impose conditions on the birth and consumption rates that will ensure that $\zeta(t)=\cos (\gamma t)$ is an explicit solution. Let us denote $\tilde{N}_{0}=\int \omega\left(a, \mu^{\prime}\right) G_{0}\left(\mu^{\prime}\right) e^{-\sigma a} d a d \mu$ and assume that $\tau\left(m \tilde{N}_{0}+x\right)=\lambda_{0}-\lambda_{1} x$ for sufficiently small $x$. Then we get

$$
\begin{aligned}
& 1+\varepsilon \cos (\gamma t)=\int b\left(a, \mu^{\prime}\right) G_{0}\left(\mu^{\prime}\right)\left(1+\varepsilon \cos \gamma\left(t-\frac{a}{\mu^{\prime}}\right)\right) e^{-\sigma a} d a d \mu^{\prime} \\
& \times\left(\lambda_{0} \tilde{N}_{0}-\lambda_{1} m \varepsilon \int \omega\left(a, \mu^{\prime}\right) G_{0}\left(\mu^{\prime}\right) \cos \gamma\left(t-\frac{a}{\mu^{\prime}}\right) e^{-\sigma a} d a d \mu^{\prime}\right) .
\end{aligned}
$$

We assume that

$$
\int b(a, \mu) G_{0}(\mu) e^{-\sigma a} \cos \left(\frac{\gamma a}{\mu}\right)=\int b(a, \mu) G_{0}(\mu) e^{-\sigma a} \sin \left(\frac{\gamma a}{\mu}\right)=0 .
$$

This leads to

$$
\begin{aligned}
& 1+\varepsilon \cos (\gamma t)=\int b(a, \mu) G_{0}(\mu) e^{-\sigma a} d a d \mu \\
& \times\left(\lambda_{0}-\lambda_{1} m \varepsilon \int \omega\left(a, \mu^{\prime}\right) G_{0}\left(\mu^{\prime}\right) \cos \left(\gamma t-\frac{\gamma a}{\mu^{\prime}}\right) e^{-\sigma a} d a d \mu^{\prime}\right) .
\end{aligned}
$$

Recall that we have

$$
1=\lambda_{0} \int b\left(a, \mu^{\prime}\right) G_{0}\left(\mu^{\prime}\right) e^{-\sigma a} d a d \mu^{\prime}
$$

so that (3.8) holds if $\lambda_{1}$ is chosen to be

$$
\lambda_{1}=-\left[m \int b(a, \mu) G_{0}(\mu) e^{-\sigma a} d a d \mu \int \omega\left(a, \mu^{\prime}\right) G_{0}\left(\mu^{\prime}\right) \cos \left(\frac{\gamma a}{\mu^{\prime}}\right) e^{-\sigma a} d a d \mu^{\prime}\right]^{-1}
$$

and

$$
\int \omega(a, \mu) G_{0}(\mu) \sin \left(\frac{\gamma a}{\mu}\right) e^{-\sigma a} d a d \mu=0 .
$$

The conditions that we have imposed in this section on functions $b, \omega$ and $\tau$ are very restrictive. However, they were imposed only to obtain explicit solutions, and existence of periodic solutions, though not of such explicit form, does not rely on these specific assumptions. This is of course related to the relation between such models and retarded differential equations which are known to lead to such kind of solutions or even to chaotic behaviors.

\subsection{The general existence of solutions and steady state}

We discuss now existence of steady states of the nonlinear Rotenberg equation

$$
\begin{aligned}
& \frac{\partial n}{\partial t}+\mu \frac{\partial n}{\partial a}+D(a, \mu) n=\int K\left(a, \mu, \mu^{\prime}\right) n\left(t, a, \mu^{\prime}\right) d \mu^{\prime} \\
& n(t, a=0, \mu)=G_{0}(\mu) \int b\left(a^{\prime}, \mu^{\prime}\right) n\left(t, a^{\prime}, \mu^{\prime}\right) d a^{\prime} d \mu^{\prime} \tau\left(\int \omega\left(a^{\prime}, \mu^{\prime}\right) n\left(t, a^{\prime}, \mu^{\prime}\right) d a^{\prime} d \mu^{\prime}\right) .
\end{aligned}
$$


We refer to $\S 3.1$ and $\S 3.2$ for the meaning of the nonlinearity $\tau$ and of $\omega$ which we assume to satisfy

$$
0 \leq \omega(a, \mu) \leq 1 \quad \tau(N) \text { decreases to } 0 \text { as } N \rightarrow \infty .
$$

A non-trivial steady state $g(a, \mu)$ must be of the form $g(a, \mu)=m G(a, \mu)$ where $G(a, \mu)$ is the solution of (see $\S 2$ for this problem)

$$
\begin{aligned}
& \mu \frac{\partial G(a, \mu)}{\partial a}+D(a, \mu) G(a, \mu)=\int K\left(a, \mu, \mu^{\prime}\right) G\left(a, \mu^{\prime}\right) d \mu^{\prime} \\
& G(a=0, \mu)=G_{0}(\mu) .
\end{aligned}
$$

The constant $m$ is the solution of

$$
\tau\left(m \int \omega(a, \mu) G(a, \mu) d a d \mu\right)=\left[\int \omega(a, \mu) G(a, \mu) d a d \mu\right]^{-1} .
$$

Solution of (3.12) exists and is unique provided that

$$
\int \omega(a, \mu) G(a, \mu) d a d \mu \geq 1 .
$$

This means that birth rate $\omega(a, \mu)$ has to be sufficiently large to overcome the competition for resource term $\tau(N)$ in the boundary condition (2.2). The main difference between the linear and nonlinear Rotenberg models is that in the non-linear case the steady state exists as long as (3.13) is satisfied, while in the linear case typically one observes either growth or decay. In particular, while large enough birth rate guarantees existence of a steady state in the nonlinear model, it leads to exponential growth in time of solutions of the linear model.

Note that solutions of the non-linear problem remain bounded in time under very mild conditions.

Proposition 3.1 Assume (2.3), (2.4), (3.10) and $0 \leq n_{0}(a, \mu) \in L^{1} \cap L^{\infty}\left(\mathbb{R}_{+} \times[0,1]\right)$. Then there exists a constant $C$ so that $0 \leq n(t, a, \mu) \leq C$ for all $t$, $a$ and $\mu, \int n(t, a, \mu) d a d \mu \leq C$ for all $t \geq 0$.

Proof. Following classical arguments (see [4] for instance) local in time non-negative solutions exist and we only need to provide a priori bounds. We show such bounds below in $L^{1} \cap L^{\infty}$. Note that since $b(a, \mu)$ and $G_{0}(\mu)$ are uniformly bounded, we have, after integrating (3.9),

$$
\frac{d N}{d t}+\sigma_{0} N \leq \int \mu n(t, a=0, \mu) d \mu \leq C \tau(N) N,
$$

where $N=\int n(t, a, \mu) d a d \mu$. This implies that there exists a constant $C=C\left(N_{0}\right)$ so that, by Gronwall's lemma, $N(t) \leq C$. This provides the uniform $L^{1}$ bound. Therefore the boundary term $n(t, a=0, \mu) \leq C$ is uniformly bounded. Hence an appropriate multiple of the constant $\left\|G_{0}\right\|_{\infty}$ is a super-solution for $n(t, a, \mu)$ (we consider that $n$ satisfies the linear equation (3.9) with an upper bound for the boundary term and initial data), and thus $n(t, a, \mu)$ is also uniformly bounded in $L^{\infty}$.

Of course, the examples of the previous subsections show that the time asymptotics cannot be simple and we further refer to [8] for examples of chaotic behaviors.

\section{Appendix}

In this section, we consider the McKendrick-Von Foerster model and we investigate its long time asymptotic behavior by means of the simple tools of multiplicators and entropy methods. This is a simpler model to explain with explicit calculations the methods used in this paper. 
We assume that the population is only age dependent and thus it is described by the density population $n(t, a) \geq 0$ with age $a \in(0, \infty)$ at time $t \in(0, \infty)$. Denoting by $b(a)$ the birth rate and $d(a)$ the death rate, the evolution of the density writes

$$
\begin{aligned}
& \frac{\partial}{\partial t} n(t, a)+\frac{\partial}{\partial a} n(t, a)+d(a) n(t, a)=0, \quad t \geq 0, a \geq 0 \\
& n(t, a=0)=\int b(a) n(t, a) d a \\
& n(t=0, a)=n_{0}(a) .
\end{aligned}
$$

On the sequel, we always make the following assumptions

$$
0 \leq d \in L_{\mathrm{loc}}^{\infty}\left(\mathbb{R}_{+}\right), \quad 0 \leq b \in M^{1}\left(\mathbb{R}_{+}\right), b \not \equiv 0
$$

Moreover, sometimes, we also make the additional assumption

$$
b \in C\left(\mathbb{R}_{+}\right) .
$$

We recall that the death rate $d(a)$ can vary drastically for different species: for trees it is often constant, for fish it decreases for small values of $a$ (smaller fish are easier targets for predators) and increases for larger $a$, for mammals it is often increasing and unbounded (Gompertz law gives an exponential growth).

Since we focus here on the qualitative properties of the solution, we merely accept that there exists a solution $n \in C\left([0, \infty) ; L^{1}(0, \infty)\right)$ to (4.1)-(4.3) for any initial datum $n_{0} \in L^{1}(0, \infty)$ and we refer to [4], [13], [15], [10], [5] for a presentation of the existence theory.

The key information to analyse the long time behavior is the so-called Malthus parameter. We define here a "generalized" Malthus parameter $\gamma \in \mathbb{R}$ by the following way

$$
\gamma:=\inf \left\{\delta \in \mathbb{R}, I(\delta)=\int_{0}^{\infty} b(a) e^{-D(a)-\delta a} d a \leq 1\right\}
$$

with the notation $D(a)=\int_{0}^{a} d\left(a^{\prime}\right) d a^{\prime}$.

Some comments are in order. The function $I(\cdot)$ is decreasing, $I(0)$ is finite, $I(\delta) \rightarrow 0$ when $\delta \rightarrow \infty$, $I(\delta) \rightarrow \infty$ when $\delta \rightarrow-\infty$, so that the expression (4.6) effectively defines a unique $\gamma \in \mathbb{R}$. Let us emphasize that, thanks to Beppo-Levi Theorem $B:=I(\gamma)$ satisfies

$$
\text { either } B=1 \quad \text { or } \quad B<1 \text { and } I(\delta)=+\infty \forall \delta<\gamma \text {. }
$$

Also remark that $B=1$ if one of the following general conditions holds: (i) $d(a) \rightarrow \infty$ as $a \rightarrow \infty$ and $b$ is bounded, (ii) $b$ is compactly supported or (iii) more generally if for some $\gamma_{0}, 1 \leq I\left(\gamma_{0}\right)<\infty$. An example when $B<1$ is a constant death rate $d(a)=d \in \mathbb{R}_{+}$and the birth rate $b(a)=\beta\left(1+a^{2}\right)^{-1}$ with $\beta \in(0,2 / \pi)$.

We also introduce the possibly infinite number

$$
A_{\dagger}:=\inf \{A>0, \operatorname{supp} b \subset[0, A]\} \in(0, \infty],
$$

which corresponds to the maximal age with which an individual can give birth. 


\subsection{Exponential growth and decay of population}

A first classical remark is that, for any constant $\bar{n}_{0}$, the function

$$
\bar{n}(t, a)=\bar{n}_{0} e^{-D(a)+\gamma(t-a)},
$$

is a particular solution of (4.1)- (4.2) if and only if, $B=1$. In that case, we easily verify that when $t \rightarrow \infty, \bar{n}(t,$.$) tends exponentially to 0$ if $\gamma<0, \bar{n}(t,$.$) tends exponentially to +\infty$ if $\gamma>0$, and $\bar{n}$ is a stationary solution when $\gamma=0$.

This function gives the long time pattern of any solution. To see this we set, for a given function $\psi$,

$$
M_{\psi}(t)=\int_{0}^{\infty} n(t, a) \psi(a) d a .
$$

Proposition 4.1 We assume (4.4), then there exists a positive function $\phi \in C_{0}\left(\mathbb{R}_{+}\right)$, supp $(\phi)=\left[0, A_{\dagger}\right]$ such that

$$
\begin{aligned}
& M_{\phi}(t)=M_{\phi}(0) e^{\gamma t} \quad \text { if } B=1, \\
& M_{\phi}(t) \leq M_{\phi}(0) e^{\gamma t} \quad \text { if } \gamma<0, B<1, \\
& M_{\phi}(t) \rightarrow 0, \quad \text { but } \quad M_{e^{D}} \nearrow \lambda_{1}<\infty \quad \text { if } \gamma=0 \text { and } B<1,
\end{aligned}
$$

For the last case (4.12) we need furthermore the additional assumption (4.5).

Since $\phi$ is positive on $\left[0, A_{\dagger}\right]$ we see that $M_{\phi}$ is a measure of the population density. Then Proposition 4.1 says that the population grows exponentially if $\gamma>0$ and decreases exponentially if $\gamma<0$. The case $\gamma=0$ and $B<1$ is interesting since the total density may be constant (for example when $d=0$ ) but the density of new generations (that $M_{\phi}$ measures) decreases. Therefore, the average age of the population increases and tends to infinity, this can be interpreted as senescence.

Proof. For a given function $\phi$, we get

$$
\frac{d}{d t} \int_{0}^{\infty} n(t, a) \phi(a) d a=\int_{0}^{\infty} n(t, a)\left(b(a) \phi(0)+\phi^{\prime}(a)-d(a) \phi(a)\right) d a .
$$

We use the function $\phi$ given by the dual problem

$$
\phi^{\prime}=(d+\gamma) \phi-\frac{b}{B}, \quad \phi(0)=1,
$$

so that

$$
\phi(a)=\frac{e^{D(a)+\gamma a}}{B} \int_{a}^{\infty} b(\alpha) e^{-D(\alpha)-\gamma \alpha} d \alpha,
$$

and $0 \leq \phi \in C\left(\mathbb{R}_{+}\right)$with $\phi(a) \rightarrow 0$ when $a \rightarrow \infty$. With this choice of $\phi$ in (4.13) we get

$$
\frac{d}{d t} M_{\phi}(t)=\gamma M_{\phi}(t)+\left(1-\frac{1}{B}\right) M_{b}(t)
$$

Assertions (4.10) and (4.11) readily follow from (4.15).

We now deal with the case $\gamma=0$ and $B<1$, which implies that $b$ is not compactly supported. First observe that thanks to $(4.15)$ we get

$$
M_{\phi} \searrow \ell \geq 0 \quad \text { and } \quad M_{b} \in L^{1}\left(\mathbb{R}_{+}\right),
$$

and we want to show that $\ell=0$. In order to prove it, we fix $T>0$ and define $n_{k}(t, a):=n\left(t_{k}+t, a\right)$ for a given sequence $\left(t_{k}\right) \nearrow+\infty$. Observing that $\inf _{(0, A)} \phi>0$ for any $A>0$ and that $\sup _{t \geq 0} M_{\phi}(t)<\infty$, 
we conclude that $\left(n_{k}\right)$ is bounded in $M^{1}((0, T) \times(0, A))$ for any $A>0$. As a consequence, up to the extraction of a subsequence, there exists $n^{*} \in M^{1}((0, T) \times(0, A))$ such that $n_{k} \rightarrow n^{*}$ in the sense of weak convergence of measure $\sigma\left(M^{1}((0, A) \times(0, T)), C([0, A] \times[0, T])\right) *$. Then, we may pass to the limit

$$
\int_{0}^{T} \int_{0}^{A} b n^{*} d a d t \leq \liminf \int_{0}^{T} \int_{0}^{A} b n_{k} d a d t \leq \liminf \int_{t_{k}}^{\infty} M_{b}(t) d t=0
$$

so that $\int_{0}^{T} \int_{0}^{\infty} b n^{*} d a d t=0$ and $n^{*}=0$ on $(0, T) \times \operatorname{supp} b$. since $n^{*}$ satisfies the transport equation (4.1), we deduce that $n^{*} \equiv 0$, which in turn implies

$$
n(t, .) \rightarrow 0 \quad \sigma\left(M^{1}(0, A), C([0, A])\right) * \text { for any } A>0 .
$$

Therefore $\ell=0$. On the other hand, choosing $\psi$ to be the solution of $\psi^{\prime}=d \psi, \psi(0)=1$ as a multiplicator in (4.13) we get

$$
\frac{d}{d t} M_{e^{D}}=M_{b} .
$$

Since $M_{b} \in L^{1}$ we deduce that $M_{e^{D}} \nearrow \lambda_{1}<\infty$. Moreover, since $e^{D} \geq 1$ and $\phi \rightarrow 0$, assertion (4.12) follows from (4.16).

We can be slightly more precise thanks to the following maximum principle.

Proposition 4.2 We assume (4.4) and $(b-d)^{+} \in L^{\infty}$. For any solutions $n_{1}$ and $n_{2}$ of (4.1)-(4.3) such that $n_{1}(0, a) \leq n_{2}(0, a) \forall a \geq 0$, there holds

$$
n_{1}(t, a) \leq n_{2}(t, a) \quad \forall t, a \geq 0 .
$$

Proof. Define $j(s)=(s)_{+}$and set $n=n_{2}-n_{1}$. By assumption, $n$ satisfies

$$
\partial_{t} n+\partial_{a} n=-d n, \quad n(t, 0)=\int_{0}^{\infty} n b d a, \quad n(0, a) \leq 0 .
$$

Multiplying that equation by $j^{\prime}(n)$ and integrating with respect to the age variable, we get

$$
\begin{aligned}
\frac{d}{d t} \int_{0}^{\infty} j(n) d a= & j\left(\int_{0}^{\infty} n b d a\right)-\int_{0}^{\infty} d n j^{\prime}(n) d a \\
& \leq\left\|(b-d)^{+}\right\|_{L^{\infty}} \int_{0}^{\infty} j(n) d a .
\end{aligned}
$$

We conclude, thanks to Gronwall lemma, that $\int_{0}^{\infty} j(n) d a=0$ and that concludes the proof.

Remark As an immediate consequence we get: If $\gamma<0$ and $0 \leq n_{0} \leq M e^{-D(a)-\gamma a}$ for some $M>0$ then

$$
0 \leq n(t, a) \leq M e^{-D(a)+\gamma(t-a)} \rightarrow 0 .
$$

If $\gamma>0$ and $n_{0} \geq m e^{-D(a)-\gamma a}$ for some $m>0$ then

$$
n(t, a) \geq m e^{-D(a)+\gamma(t-a)} \rightarrow \infty .
$$

By regularization arguments we may even go further and prove that there exists at least a solution which satisfies the above conclusion without the assumption $(b-d)^{+} \in L^{\infty}$. 


\subsection{Equilibrium case}

We now focus on the case $\gamma=0$ and $B=1$. Remark that, defining the new density $\tilde{n}(t, a):=n(t, a) e^{\gamma t}$, we are reduced to this case under the assumption $B=1$.

Proposition 4.3 We assume (4.4), $\int b(a) e^{D(a)} d a=1, b>0$ a.e. on some interval $] a_{0}, a_{1}[$. Then, for any $n_{0} \in L^{\infty}\left(0, A_{\dagger}\right)$ the solution $n(t,$.$) satisfies$

$$
n(t, .) \underset{t \rightarrow \infty}{\longrightarrow} n^{*} e^{-D}, \quad \text { in } L_{\mathrm{loc}}^{1}\left(\mathbb{R}_{+}\right),
$$

with $n^{*}$ the constant uniquely determined thanks to the conservation law

$$
M_{\phi}(t)=M_{\phi}(0)=n^{*} \int_{0}^{\infty} e^{-D} d a, \quad \phi^{\prime}=d \phi-b, \quad \phi(0)=1 .
$$

If additionally, we assume that for some $\mu>0$, we have be $e^{-D} \geq \mu \phi$, then

$$
\int_{0}^{\infty}\left|n(t, a)-n^{*} e^{-D}\right| d a \leq e^{-\mu t} \int_{0}^{\infty}\left|n(0, a)-n^{*} e^{-D}\right| d a .
$$

Remarks 1. Concerning the assumption on the positivity of $b$, we recall that for $b=\delta\left(a=a_{\dagger}\right)$ (Dirac mass), there are periodic solutions and the conclusion of the theorem is wrong

2. We recall that an exponential rate of convergence can be proved under more general assumptions using Laplace transform (see the above references). We present this new method here in order to show on a simple example the arguments used for Rotenberg model.

Proof. We first prove the generic convergence result. Fix a convex function $j: \mathbb{R}_{+} \rightarrow \mathbb{R}_{+}$such that $j(0)=0$. A direct computation shows that

$$
\frac{\partial}{\partial t}\left(\phi j\left(n e^{D}\right) e^{-D}\right)+\frac{\partial}{\partial a}\left(\phi j\left(n e^{D}\right) e^{-D}\right)=-b j\left(n e^{D}\right) e^{-D} .
$$

Therefore, defining the entropy functional $J$ and the entropy dissipation term $\mathcal{J}$ by

$$
\begin{gathered}
J(n):=\int_{0}^{\infty} \phi j\left(n e^{D}\right) e^{-D} d a \\
\mathcal{J}(n):=\int_{0}^{\infty} j\left(n e^{D}\right) b e^{-D} d a-j\left(\int_{0}^{\infty}\left(n e^{D}\right) b e^{-D} d a\right) \geq 0,
\end{gathered}
$$

the following H-Theorem holds

$$
\frac{d}{d t} J(n(t, .))=-\mathcal{J}(n(t, .)) \leq 0
$$

For any given sequence $\left(t_{k}\right) \nearrow+\infty$, we define $n_{k}(t, a)=n\left(t+t_{k}, a\right)$. Thanks to the H-Theorem we have

$$
\int_{0}^{\infty}\left\{j\left(\int_{0}^{\infty}\left(n_{k} e^{D}\right) b e^{-D} d a\right)-\int_{0}^{\infty} j\left(n_{k} e^{D}\right) b e^{-D} d a\right\} d t \rightarrow 0
$$

which implies, after extracting subsequences (recall that $n$ is uniformly bounded thanks to Proposition $4.2)$,

$$
n_{k^{\prime}} \rightarrow \bar{n}, \quad \text { with } \quad \bar{n}=\varphi(t) e^{-D} \quad b \text {-a.e. } \mathbb{R}_{+} \times \mathbb{R}_{+} .
$$

Since, on $\left.\mathbb{R}_{+} \times\right] a_{0}, a_{1}[$, there holds

$$
0=\partial_{t} \bar{n}+\partial_{a} \bar{n}-d \bar{n}=\varphi^{\prime}(t) e^{-D}
$$


so that $\varphi(t)=\varphi_{0}$ is a constant. Using now that the equation is satisfied by $\bar{n}$ on $\mathbb{R}_{+} \times \mathbb{R}_{+}$, we get $\bar{n}(t, a)=\varphi_{0} e^{-D}$ (this requires a simple truncation argument when $A_{\dagger}=\infty$ ). Thanks to the conservation laws established in the proof of Proposition 4.1 we may identify $\varphi_{0}=n^{*}$. By uniqueness, we conclude that the full family

$$
n\left(t+t_{k}, a\right) \underset{k \rightarrow \infty}{\rightarrow} n^{*} e^{-D(a)} \quad L^{\infty}\left(\mathbb{R}_{+} \times \mathbb{R}_{+}\right)-w .
$$

It yields

$$
n\left(t+t_{k}, 0\right)=\int_{0}^{A} b n(t, a) d a \underset{k \rightarrow \infty}{\rightarrow} n^{*} \quad L^{\infty}\left(\mathbb{R}_{+}\right)-w .
$$

On the other hand, for any $\varphi \in C_{c}^{1}(0, A)$, there holds

$$
\frac{d}{d t} \int_{0}^{A} n(t, a) \varphi d a=\int_{0}^{A}\left(d+\varphi^{\prime}\right) n d a \in L^{\infty}\left(\mathbb{R}_{+}\right),
$$

so that, by standard arguments, for all $T>0$,

$$
n\left(t+t_{k}, 0\right) \underset{k \rightarrow \infty}{\rightarrow} n^{*} \quad C([0, T]) .
$$

Writing

$$
\frac{d}{d t} \int_{0}^{A}|n-\bar{n}|\left(t+t_{k}, a\right) d a=\left|n\left(t+t_{k}, 0\right)-\bar{n}\left(t+t_{k}, 0\right)\right| d a,
$$

we conclude that

$$
\sup _{[0, T]} \int_{0}^{A}|n-\bar{n}|\left(t+t_{k}, a\right) d a \leq T \sup _{[0, T]}\left|n\left(t+t_{k}, 0\right)-n^{*}\right| \rightarrow 0,
$$

and the first asymptotic result is proved.

We now prove the exponential decay. First, by linearity, we can replace $n$ by $n-n^{*} e^{-D}$ in the equation and thus $\int_{0}^{\infty} n(t, a) \phi(a) d a=0$. Then, using $j(n)=|n|$ we find

$$
\begin{gathered}
\frac{d}{d t} \int_{0}^{\infty} \phi|n(t)| d a=-\int_{0}^{\infty} b(a)|n(t)| d a+\left|\int_{0}^{\infty} b(a) n(t) d a\right| \\
=-\int_{0}^{\infty} b(a)|n(t)| d a+\left|\int_{0}^{\infty}(b(a)-\mu \phi) n(t) d a\right| \\
\leq-\int_{0}^{\infty} b(a)|n(t)| d a+\int_{0}^{\infty}(b(a)-\mu \phi)|n(t)| d a \\
=-\mu \int_{0}^{\infty} \phi|n(t)| d a .
\end{gathered}
$$

And we conclude again by Gronwall lemma.

\section{References}

[1] R. Arking, Biology of Aging. Sinauer, 1998.

[2] C. Bardos, R. Santos and R. Sentis, Diffusion approximation and computation of the critical size. Trans. Amer. Math. Soc. 284 (1984), no. 2, 617-649. 
[3] A. Bensoussan, J.-L. Lions, and G. Papanicolaou, Boundary layers and homogenization of transport processes, RIMS, Kyoto Univ., 15, 1979, 53-157.

[4] M. Chipot, On the equations of age-dependent population dynamics. Arch. Rational Mech. Anal. 82 (1983), no. 1, 13-25.

[5] J.M. Cushing, An Introduction to Structured Population Dynamics. CBMS-NSF, Regional conference series in applied mathematics, SIAM 1998

[6] R. Dautray and J.-L. Lions, Analyse Mathématique et cacul numérique pour les sciences et les techniques. Masson, 1988.

[7] O. Diekmann, M. Gyllenberg, J. A. J. Metz, Steady state analysis of structured population models. Preprint 2002.

[8] J. Dyson, R. Villella-Bressan and G. Webb, A nonlinear age and maturity structured model of population dynamics. II. Chaos. J. Math. Anal. Appl. 242 (2000), no. 2, 255-270.

[9] L. Edelstein-Keshet, A. Israel and P. Lansdorp, Modelling perspectives on aging: can mathematics help us stay young? J. Theor. Biol. 213 (2001) 509-525.

[10] M. Iannelli, Mathematical Theory of Age-Structured Population Dynamics. Applied Mathematics Monographs 7, Giardini Editori e Stampatori, Pisa 1994

[11] J.L. Lebowitz and S.I. Rubinow, A theory for the age and generation time distribution of a microbial population. J. Math. Biology 1 (1977) 17-36.

[12] P. Marcati, Asymptotic behavior in age-dependent population dynamics with hereditary renewal law, SIAM J. Math. Anal. Vol. 12, No 6 (1981) 904-916.

[13] J. A. J. Metz and O. Diekmann, the dynamics of physiologically structured populations. LN in biomathematics 68, Springer-Verlag, 1986.

[14] J.D. Murray, Mathematical biology. Springer-Verlag, 1993.

[15] G. F. Webb, Theory of nonlinear age-dependent population dynamics. M. Dekker, New-York, 1985.

[16] J. S. Weitz and H. B. Fraser, Explaining mortality rate plateaus, PNAS Vol. 98, No 26, 1538315386 (2001).

[17] M. Rotenberg, Transport theory for growing cell populations. J. Theor. biology 103 (1983) 181-199. 